\title{
Perturbative solutions of the $f(R)$-theory of gravity in a central gravitational field and some applications
}

\author{
Nguyen Anh Ky ${ }^{1,2, a}$, Pham Van Ky ${ }^{3, b}$, Nguyen Thi Hong Van ${ }^{2,4, c}$ \\ ${ }^{1}$ Duy Tan University, K7/25 Quang Trung Street, Hai Chau, Da Nang, Vietnam \\ ${ }^{2}$ Institute of Physics, Vietnam Academy of Science and Technology, 10 Dao Tan, Ba Dinh, Hanoi, Vietnam \\ ${ }^{3}$ Graduate University of Science and Technology, Vietnam Academy of Science and Technology, 18 Hoang Quoc Viet, Cau Giay, Hanoi, Vietnam \\ ${ }^{4}$ Institute for Interdisciplinary Research in Science and Education, ICISE, Quy Nhon, Vietnam
}

Received: 25 March 2018 / Accepted: 23 June 2018 / Published online: 30 June 2018

(C) The Author(s) 2018, corrected publication August 2018

\begin{abstract}
Exact solutions of an $f(R)$-theory (of gravity) in a static central (gravitational) field have been studied in the literature quite well, but, to find and study exact solutions in the case of a non-static central field are not easy at all. There are, however, approximation methods of finding a solution in a central field which is not necessarily static. It is shown in this article that an approximate solution of an $f(R)$ theory in a general central field, which is not necessary to be static, can be found perturbatively around a solution of the Einstein equation in the general theory of relativity. In particular, vacuum solutions are found for $f(R)$ of general and some special forms. Further, applications to the investigation of a planetary motion and light's propagation in a central field are presented. An effect of an $f(R)$-gravity is also estimated for the SgrA*-S2 system. The latter gravitational system is much stronger than the Sun-Mercury system, thus the effect could be much stronger and, thus, much more measurable.
\end{abstract}

\section{Introduction}

The General theory of Relativity (GR) $[1,2]$ announced in 1915 was theoretically developed by A. Einstein, and it has been experimentally verified as an excellent theory of gravitation. In particular, the GR was once again confirmed triumphantly by recent detections of gravitational waves (see, for example, $[3,4])$. The GR is governed by the Einstein equation $[1,2,5]$

$$
R_{\mu \nu}-\frac{1}{2} R g_{\mu \nu}=-\frac{8 \pi G}{c^{4}} T_{\mu \nu}
$$

\footnotetext{
a e-mail: anhky@iop.vast.ac.vn

b e-mail: phamkyvatly@gmail.com

c e-mail: nhvan@iop.vast.ac.vn
}

obtained from the Lagrangian $\mathcal{L}_{G}=R$. This equation can describe very well gravitational phenomena of the normal matter, but it ineffectively describes other phenomena such as the Universe's accelerated expansion (supposed to be explained by the introduction of the concept of the socalled dark energy or cosmological constant), dark matter, cosmic inflation, quantum gravity, etc. One of the simplest suggestions for solving the dark energy problem is adding the cosmological constant $\Lambda$ to the Lagrangian, that is, $\mathcal{L}_{G}=R-2 \Lambda$, leading to the equation of motion $[1,5]$

$R_{\mu \nu}-\frac{1}{2} R g_{\mu \nu}+\Lambda g_{\mu \nu}=-\frac{8 \pi G}{c^{4}} T_{\mu \nu}$.

According to the latter equation, the Universe would acceleratedly expand. However, there is also room for doubt in this case (see [6-8] for more discussions).

A more general theory, ${ }^{1}$ which can be used to solve the above-mentioned problems and explain some other phenomena in cosmology is that with Lagrangian $\mathcal{L}_{G}=f(R)$, where $f(R)$ is a scalar function of the scalar curvature $R$. The equation of motion now becomes [7-9]

$$
\begin{array}{r}
f^{\prime}(R) R_{\mu \nu}-g_{\mu \nu} \square f^{\prime}(R)+\nabla_{\mu} \nabla_{\nu}, f^{\prime}(R)-\frac{1}{2} f(R) g_{\mu \nu} \\
=-k T_{\mu \nu},
\end{array}
$$

where $k=\frac{8 \pi G}{c^{4}}, \square=\nabla_{\mu} \nabla^{\mu}$ and $\nabla_{\mu}$ is the covariant derivative. This theory is called $f(R)$-theory of gravity or just $f(R)$-gravity or $f(R)$-theory for short. Nowadays, this theory is becoming a hot topical issue and attracting much interest of many cosmologists (for review, see, for example,

\footnotetext{
1 There are also other models extending the GR, however, they are not in the scope of the present paper (see [7,8] and references therein, for listing some of them).
} 
[7-13]). There are a lot of variants of the $f(R)$-theory such as those with $f(R)=R+\lambda R^{2}$ or $f(R)=R-\frac{\lambda}{R^{n}}$, etc., each them can explain some of the cosmological phenomena but none of them is perfect [7-16]. In general, to find a solution, especially, an exact one, of an $f(R)$-theory is very difficult, even impossible. To simplify the situation, some reasonable conditions are sometimes imposed so that approximate solutions can be found. One of such conditions could be that of a spherical symmetry which is a quite good approximation in many cases. Here, a general $f(R)$-theory will be considered in a spherically symmetric (gravitational) field called usually a central field.

Exact solutions of the $f(R)$-theory in a static central field are studied in [17-22] but there are also approximation methods for central fields which are not necessarily static [23-25]. In this article, approximate solutions of the $f(R)$-theory for a general and some special cases in a general central field are found by perturbation around the Einstein equation. Then, we can use the obtained solutions to calibrate parameters of orbits of planets.

In this article the following conventions are used:

- Metric signature in Minkowski space: $(+,-,-,-)$, that is, the infinitesimal distance is calculated as

$$
\begin{aligned}
d s^{2} & =\eta_{\mu \nu} d x^{\mu} d x^{\nu}=d x^{0} d x_{0}+d x^{i} d x_{i}, \\
& =c^{2} d t^{2}-d x^{2}-d y^{2}-d z^{2},
\end{aligned}
$$

with Latin letters used for three-dimensional spatial indices, and, Greek letters used for four-dimensional space-time indices.

- Riemann curvature tensor:

$$
R_{\mu \beta \nu}^{\alpha}=\frac{\partial \Gamma_{\mu \beta}^{\alpha}}{\partial x^{\nu}}-\frac{\partial \Gamma_{\mu \nu}^{\alpha}}{\partial x^{\beta}}+\Gamma_{\sigma \nu}^{\alpha} \Gamma_{\mu \beta}^{\sigma}-\Gamma_{\sigma \beta}^{\alpha} \Gamma_{\mu \nu}^{\sigma} .
$$

- Rank-2 curvature tensor (Ricci tensor): $R_{\mu \nu}=R_{\mu \alpha \nu}^{\alpha}$.

- Scalar curvature: $R=g^{\mu \nu} R_{\mu \nu}$.

- Energy-momentum tensor of a macroscopic object:

$$
T_{\mu \nu}=\frac{1}{c^{2}}(\varepsilon+p) u_{\mu} u_{\nu}-p g_{\mu \nu},
$$

where $u^{\mu}=\frac{d x^{\mu}}{d \tau}=c \frac{d x^{\mu}}{d s}$, while $\varepsilon$ and $p$ are the energy density and the pressure, respectively.

In the next section we will consider a general $f(R)$-theory in a central field and its perturbative solutions. In particular, solutions in vacuum are also investigated for a general form and some special forms of $f(R)$. Section 3 is devoted to applications of the obtained solutions to investigating a planet's and light's motion in a central field. Some comments and conclusion will be made in Sect. 4. Finally, a proof of formula (37) is exposed in the appendix.

\section{$2 f(R)$-theory and perturbative solutions}

Now we consider a system of matter in a gravitational field. If the gravitational field's Lagrangian is $\mathcal{L}_{G}=R$ and the matter Lagrangian is $\mathcal{L}_{M}$, the system's action has the form

$$
\begin{aligned}
S & =S_{G}+S_{M}, \\
& =\frac{c^{3}}{16 \pi G} \int R \sqrt{-g} d^{4} x+\frac{1}{c} \int \mathcal{L}_{M} \sqrt{-g} d^{4} x .
\end{aligned}
$$

The Einstein equation obtained from this action $[1,2]$ is

$R_{\mu \nu}-\frac{1}{2} g_{\mu \nu} R=-\frac{8 \pi G}{c^{4}} T_{\mu \nu}$,

where $T_{\mu \nu}$ is the energy-momentum tensor of matter

$$
\begin{aligned}
T_{\mu \nu} & :=\frac{+2}{\sqrt{-g}} \frac{\delta S_{M}}{\delta g^{\mu \nu}}, \\
& =-\mathcal{L}_{M} g_{\mu \nu}+2 \frac{\partial \mathcal{L}_{M}}{\partial g^{\mu \nu}}-\frac{2}{\sqrt{-g}} \frac{\partial}{\partial x^{\alpha}}\left(\frac{\partial\left(\mathcal{L}_{M} \sqrt{-g}\right)}{\partial \frac{\partial g^{\mu \nu}}{\partial x^{\alpha}}}\right)
\end{aligned}
$$

Taking a trace of (2), we get

$R=\frac{8 \pi G}{c^{4}} T$,

with $T=T_{\mu}^{\mu}$, and the Eq. (2) becomes

$R_{\mu \nu}=-\frac{8 \pi G}{c^{4}}\left(T_{\mu \nu}-\frac{1}{2} g_{\mu \nu} T\right)$.

For $\mathcal{L}_{G}=f(R)$, the system's action is

$$
\begin{aligned}
S & =S_{G}+S_{M}, \\
& =\frac{c^{3}}{16 \pi G} \int f(R) \sqrt{-g} d^{4} x+\frac{1}{c} \int \mathcal{L}_{M} \sqrt{-g} d^{4} x,
\end{aligned}
$$

leading to the equation of motion [7-9]

$$
\begin{array}{r}
f^{\prime}(R) R_{\mu \nu}-g_{\mu \nu} \square f^{\prime}(R)+\nabla_{\mu} \nabla_{\nu} f^{\prime}(R)-\frac{1}{2} f(R) g_{\mu \nu} \\
=-k T_{\mu \nu} .
\end{array}
$$

If the $f(R)$-theory deffers from the Einstein theory (when $f(R)=R$ ) just slightly, we can write $f(R)$ in the form

$f(R)=R+\lambda h(R)$, 
where $h(R)$ is a scalar function of $R$ and $\lambda$ is a parameter such that $\lambda h(R)$ and its derivatives compared with $R$ are a very small. Substituting (7) into (6) we obtain

$$
\begin{array}{r}
{\left[1+\lambda h^{\prime}(R)\right] R_{\mu \nu}-g_{\mu \nu} \square\left[\lambda h^{\prime}(R)\right]+\nabla_{\mu} \nabla_{\nu}\left[\lambda h^{\prime}(R)\right]} \\
-\frac{1}{2} g_{\mu \nu}[R+\lambda h(R)]=-k T_{\mu \nu},
\end{array}
$$

or

$$
\begin{array}{r}
R_{v}^{\mu}-\frac{1}{2} \delta_{\nu}^{\mu} R+\lambda h^{\prime}(R) R_{v}^{\mu}-\frac{\lambda}{2} \delta^{\mu}{ }_{v} h(R)-\lambda \delta^{\mu}{ }_{v} \square h^{\prime}(R) \\
+\lambda \nabla^{\mu} \nabla_{\nu} h^{\prime}(R)=-k T_{v}^{\mu} .
\end{array}
$$

We solve the latter equation by a perturbation method basing on the fact that this equation differs from the Einstein equation by small perturbative terms (the last four terms on the left-hand side of the last equation). Substituting a solution of the Einstein equation, i.e., (4) and (5),

$R=k T, \quad R_{v}^{\mu}=-k\left(T_{v}^{\mu}-\frac{1}{2} \delta^{\mu}{ }_{\nu} T\right)$,

into the perturbative terms in Eq. (8),

$$
\begin{array}{r}
R_{v}^{\mu}-\frac{1}{2} \delta^{\mu}{ }_{v} R-\lambda k h^{\prime}(k T)\left(T^{\mu}{ }_{v}-\frac{1}{2} \delta^{\mu}{ }_{v} T\right)-\frac{\lambda}{2} \delta^{\mu} h(k T) \\
-\lambda \delta^{\mu}{ }_{v} \square^{E} h^{\prime}(k T)+\lambda \nabla^{\mu} \nabla_{v}^{E} h^{\prime}(k T)=-k T_{v}^{\mu},
\end{array}
$$

we solve the latter perturbatively at the first order, where $h^{\prime}(k T)=\frac{\partial h(k T)}{\partial(k T)}$ and the superscript $E$ in the covariant differentiations means that the metric tensor $g_{\mu \nu}$ is taken in the Einstein equation solutions. If we solve the above equation in vacuum $\left(T_{v}^{\mu}=0, T=0\right)$, then $h(k T)$ and $h^{\prime}(k T)$ are constants, hence their differentiations are equal to zero, the Eq. (9) becomes

$R^{\mu}{ }_{v}-\frac{1}{2} \delta^{\mu}{ }_{v} R=\frac{\lambda}{2} \delta^{\mu}{ }_{\nu} h(k T)=\frac{\lambda}{2} \delta^{\mu}{ }_{\nu} h(0)$.

Note: If $h(0)=0$ the perturbative equation (10) is similar to the Einstein equation in vacuum. But, there is a fundamental difference. With the Einstein equation in a central field, a solution in vacuum is stationary and determined upto a constant (of time) even when the central field is not stationary. In a spherically symmetric $f(R)$-theory, however, as seen later, a solution in general is not stationary. Furthermore, the integration constant in the solution of the Einstein equation can be found by taking a limit at the classical gravitational potential $\varphi=-\frac{G M}{r}$, but a similar step cannot be done with the $f(R)$-theory as the classical gravitational potential may be different from $\varphi=-\frac{G M}{r}$, though little. Hence, we will solve Eq. (9) in a general way, not only in vacuum.
We are using a spherically symmetric metric in the shape of the Schwarzschild metric [2],

$d s^{2}=e^{u(r, t)} d x^{o 2}-e^{v(r, t)} d r^{2}-r^{2}\left(d \theta^{2}+\sin ^{2} \theta d \varphi^{2}\right)$,

with the following non-zero metric elements

$$
\begin{gathered}
g_{00}=e^{u(r, t)}, g_{11}=-e^{v(r, t)}, \\
g_{22}=-r^{2}, g_{33}=-r^{2} \sin ^{2} \theta,
\end{gathered}
$$

(writing $g_{00}=e^{u(r, t)}$ does not mean it always positive because $u(r, t)$ can be complex). With the given metric we can calculate any element of the Ricci tensor [2], say $R_{0}^{1}$,

$R_{0}^{1}=\frac{e^{-v(r, t)}}{r} \frac{\partial v(r, t)}{\partial c t}$,

which, when inserted in Eq. (6) for vacuum, gives

$f^{\prime}(R) \frac{e^{-v(r, t)}}{r} \frac{\partial v(r, t)}{\partial c t}=-\nabla^{1} \nabla_{0} f^{\prime}(R)$.

Considering the case of a central gravitational field, we see that the Einstein theory in vacuum, as well known, is always stationary, but, a general $f(R)$-theory, with $f(R) \neq R$, is not stationary in vacuum. It can be seen from the fact that the right-hand side of (13) in general is not zero, therefore, $\frac{\partial v(r, t)}{\partial t} \neq 0$, thus, the metric may varies with time. However, the non-stationarity does not show up at the first order of perturbation by using $R_{0}^{1}$ in the approximate equation (9). To see the non-stationarity appearing at the first order of perturbation, it is enough to use $R_{0}^{0}$ and $R_{1}^{1}$ in the latter equation.

With the metric (11) we get [2]

$R_{0}^{0}-\frac{1}{2} R=e^{-v(r, t)}\left[\frac{1}{r^{2}}-\frac{v^{\prime}(r, t)}{r}\right]-\frac{1}{r^{2}}$,

where $v^{\prime}(r, t)=\frac{\partial v(r, t)}{\partial r}$. Comparing (14) with (9) we obtain

$$
\begin{aligned}
& e^{-v(r, t)} {\left[\frac{1}{r^{2}}-\frac{v^{\prime}(r, t)}{r}\right]-\frac{1}{r^{2}}=-k T_{0}^{0}+\frac{\lambda}{2} h(k T) } \\
&+\lambda \nabla^{i} \nabla_{i}^{E} h^{\prime}(k T)+\lambda k\left(T_{0}^{0}-\frac{T}{2}\right) h^{\prime}(k T),
\end{aligned}
$$

where $\nabla^{i} \nabla_{i}=\square-\nabla^{0} \nabla_{0}$, or

$$
\begin{array}{r}
e^{-v(r, t)} \frac{v^{\prime}(r, t)}{r}+\frac{1}{r^{2}}\left[1-e^{-v(r, t)}\right]=k T_{0}^{0}-\frac{\lambda}{2} h(k T) \\
-\lambda \nabla^{i} \nabla_{i}^{E} h^{\prime}(k T)-\lambda k\left(T_{0}^{0}-\frac{T}{2}\right) h^{\prime}(k T) .
\end{array}
$$


If we write $v(r, t)$ in the form

$v(r, t)=-\ln \left[1+\frac{c(r, t)}{r}\right]$,

we get from (15)

$$
\begin{array}{r}
-\frac{c^{\prime}(r, t)}{r^{2}}=k T_{0}^{0}-\frac{\lambda}{2} h(k T)-\lambda \nabla^{i} \nabla_{i}^{E} h^{\prime}(k T) \\
-\lambda k\left(T_{0}^{0}-\frac{T}{2}\right) h^{\prime}(k T) .
\end{array}
$$

Integrating the latter equation

$$
\begin{array}{r}
c(r, t)=-\int_{0}^{r}\left[k T_{0}^{0}-\frac{\lambda}{2} h(k T)-\lambda \nabla^{i} \nabla_{i}^{E} h^{\prime}(k T)\right. \\
\left.\quad-\lambda k\left(T_{0}^{0}-\frac{T}{2}\right) h^{\prime}(k T)\right] r^{\prime 2} d r^{\prime},
\end{array}
$$

and substituting the result (17) into (16), we obtain

$$
\begin{array}{r}
v(r, t)=-\ln \left\{1-\frac{1}{r} \int_{0}^{r}\left[k T_{0}^{0}-\frac{\lambda}{2} h(k T)\right.\right. \\
\left.\left.-\lambda \nabla^{i} \nabla_{i}^{E} h^{\prime}(k T)-\lambda k\left(T_{0}^{0}-\frac{T}{2}\right) h^{\prime}(k T)\right] r^{\prime 2} d r^{\prime}\right\},
\end{array}
$$

where $T_{0}^{0}=T_{0}^{0}\left(r^{\prime}, t\right)$ and $T=T\left(r^{\prime}, t\right)$.

Let us now calculate the integrand $\nabla^{i} \nabla_{i}^{E} h^{\prime}(k T)$ in (18). Because of the spherical symmetry, $T$, thus, $h^{\prime}(k T)$ does not depend on $\theta$ and $\varphi$, but $r$ and $t$ only. Putting only nonvanishing elements of $g_{\mu \nu}$ and $\Gamma_{\mu \nu}^{\alpha}$ in the intergrand, we have

$$
\begin{array}{r}
\nabla^{i} \nabla_{i} h^{\prime}(k T)=g^{11} \partial_{1} \partial_{1} h^{\prime}(k T)-g^{11} \Gamma_{11}^{0} \partial_{o} h^{\prime}(k T) \\
-g^{i j} \Gamma_{i j}^{1} \partial_{1} h^{\prime}(k T),
\end{array}
$$

here $\partial_{0}=\frac{\partial}{\partial x^{0}}=\frac{\partial}{\partial c t}, \partial_{1}=\frac{\partial}{\partial r}$ and $g^{i j} \Gamma_{i j}^{1}=g^{11} \Gamma_{11}^{1}+$ $g^{22} \Gamma_{22}^{1}+g^{33} \Gamma_{33}^{1}$. On the other hand, also because of the spherical symmetry, we have

$$
\begin{aligned}
g^{11} \Gamma_{11}^{0} & =-\frac{g^{11} g^{00}}{2} \frac{\partial g_{11}}{\partial x^{0}}=-\frac{g^{11} g^{00}}{2} \frac{\partial \frac{1}{g^{11}}}{\partial x^{0}}=\frac{1}{2 c} \frac{g^{00}}{g^{11}} \frac{\partial g^{11}}{\partial t}, \\
g^{i j} \Gamma_{i j}^{1} & =g^{11} g^{11} \frac{\partial g_{11}}{\partial x^{1}}-\frac{1}{2} g^{11} g^{i i} \frac{\partial g_{i i}}{\partial x^{1}} \\
& =g^{11} g^{11} \frac{\partial \frac{1}{g^{11}}}{\partial x^{1}}-\frac{1}{2} g^{11} g^{i i} \frac{\partial g_{i i}}{\partial x^{1}} \\
& =-\frac{1}{2} \frac{\partial g^{11}}{\partial r}-\frac{2}{r} g^{11} .
\end{aligned}
$$

Finally, substitutions of (20) and (21) into (19) give

$$
\begin{array}{r}
\nabla^{i} \nabla_{i}^{E} h^{\prime}(k T)=-\frac{1}{2 c^{2}} \frac{g_{E}^{00}}{g_{E}^{11}} \frac{\partial g_{E}^{11}}{\partial t} \frac{\partial h^{\prime}(k T)}{\partial t}+g_{E}^{11} \frac{\partial^{2} h^{\prime}(k T)}{\partial r^{2}} \\
+\left(\frac{2}{r} g_{E}^{11}+\frac{1}{2} \frac{\partial g_{E}^{11}}{\partial r}\right) \frac{\partial h^{\prime}(k T)}{\partial r} .
\end{array}
$$

Here, as said before, the subscript $E$ indicates the Einstein limit.

\subsection{Vacuum solutions}

Now we consider solutions in vacuum for a body-gravitation source of radius $R_{0}$ which in general depends on time, $R_{0}=$ $R_{0}(t)$. Because of considering solutions in vacuum, we can neglect the pressure. It means that the tensor $T$ has $T_{0}^{0}$ as the only non-zero component, and Eq. (18) becomes

$$
\begin{aligned}
v(r, t)= & -\ln \left\{1-\frac{1}{r} \int_{0}^{r}\left[k T_{0}^{0}-\frac{\lambda}{2} h\left(k T_{0}^{0}\right)\right.\right. \\
& \left.\left.-\frac{\lambda}{2} k T_{0}^{0} h^{\prime}\left(k T_{0}^{0}\right)-\lambda \nabla^{i} \nabla_{i}^{E} h^{\prime}\left(k T_{0}^{0}\right)\right] r^{\prime 2} d r^{\prime}\right\},
\end{aligned}
$$

with $\nabla^{i} \nabla_{i}^{E} h^{\prime}\left(k T_{0}^{0}\right)$ calculated by (22). As $T_{0}^{0}=0$ in vacuum, the first integration in (23) spreads only between 0 and $R_{0}(t)$ and gives

$\int_{0}^{R_{0}(t)} k T_{0}^{0}\left(r^{\prime}, t\right) r^{\prime 2} d r^{\prime}=\frac{k M c^{2}}{4 \pi}$

with $M$ being the mass of the body-gravitation source. Assuming that the body-gravitation source is uniform we have

$M=\frac{4}{3} \pi\left[R_{0}(t)\right]^{3} \times \rho$,

where, $\rho$ is the mass density which is independent from coordinates, and, thus,

$T_{0}^{0}=\frac{M c^{2}}{\frac{4}{3} \pi\left[R_{0}(t)\right]^{3}}$.

Putting (24) in (23) we get

$$
\begin{aligned}
v(r, t)= & -\ln \left\{1-\frac{k c^{2} M}{4 \pi r}+\frac{\lambda}{r} \int_{0}^{r}\left[\frac{1}{2} h\left(k T_{0}^{0}\right)\right.\right. \\
& \left.\left.+\frac{1}{2} k T_{0}^{0} h^{\prime}\left(k T_{0}^{0}\right)+\nabla^{i} \nabla_{i}^{E} h^{\prime}\left(k T_{0}^{0}\right)\right] r^{\prime 2} d r^{\prime}\right\} .
\end{aligned}
$$


Next we calculate $u(r, t)$ in $g_{00}(r, t)$. Considering (9) in vacuum, see (10),

$R_{0}^{0}-\frac{1}{2} R=R_{1}^{1}-\frac{1}{2} R$,

and using the metric (11), we have [2]

$R_{0}^{0}-\frac{1}{2} R=-e^{-v(r, t)}\left[\frac{v^{\prime}(r, t)}{r}-\frac{1}{r^{2}}\right]-\frac{1}{r^{2}}$,

$R_{1}^{1}-\frac{1}{2} R=e^{-v(r, t)}\left[\frac{u^{\prime}(r, t)}{r}+\frac{1}{r^{2}}\right]-\frac{1}{r^{2}}$,

and, thus, $u(r, t)=-v(r, t)$. Therefore, in vacuum, $u(r, t)$ takes the form

$$
\begin{aligned}
u(r, t)= & \ln \left\{1-\frac{k c^{2} M}{4 \pi r}+\frac{\lambda}{r} \int_{0}^{r}\left[\frac{1}{2} h\left(k T_{0}^{0}\right)\right.\right. \\
& \left.\left.+\frac{1}{2} k T_{0}^{0} h^{\prime}\left(k T_{0}^{0}\right)+\nabla^{i} \nabla_{i}^{E} h^{\prime}\left(k T_{0}^{0}\right)\right] r^{\prime 2} d r^{\prime}\right\} .
\end{aligned}
$$

In conclusion, starting from $\mathcal{L}_{G}=f(R)=R+\lambda h(R)$ and the Schwarzschild metric [thus (11), (27) and (31)], we obtain a perturbative solution in vacuum

$$
\begin{aligned}
g_{00}(r, t)= & 1-\frac{k c^{2} M}{4 \pi r}+\frac{\lambda}{r} \int_{0}^{r}\left[\frac{1}{2} h\left(k T_{0}^{0}\right)\right. \\
& \left.+\frac{1}{2} k T_{0}^{0} h^{\prime}\left(k T_{0}^{0}\right)+\nabla^{i} \nabla_{i}^{E} h^{\prime}\left(k T_{0}^{0}\right)\right] r^{\prime 2} d r^{\prime} \\
g_{11}(r, t)= & -\left\{1-\frac{k c^{2} M}{4 \pi r}+\frac{\lambda}{r} \int_{0}^{r}\left[\frac{1}{2} h\left(k T_{0}^{0}\right)\right.\right. \\
& \left.\left.+\frac{1}{2} k T_{0}^{0} h^{\prime}\left(k T_{0}^{0}\right)+\nabla^{i} \nabla_{i}^{E} h^{\prime}\left(k T_{0}^{0}\right)\right] r^{\prime 2} d r^{\prime}\right\}^{-1}, \\
g_{22}= & -r^{2}, \\
g_{33}= & -r^{2} \sin ^{2} \theta
\end{aligned}
$$

with $k=\frac{8 \pi G}{c^{4}}, T_{0}^{0}=T_{0}^{0}\left(r^{\prime}, t\right), h^{\prime}\left(k T_{0}^{0}\right)=\frac{\partial h\left(k T_{0}^{0}\right)}{\partial\left(k T_{0}^{0}\right)}$ and $\nabla^{i} \nabla_{i}^{E} h^{\prime}\left(k T_{0}^{0}\right)$ calculated in (22). Far away from the bodygravitation source $T_{0}^{0}$ can be considered depending on the time $t$ only (at a long distance, the density of the bodygravitation source can be considered homogeneous), that means the last two terms of (22) vanishing (see more details in the appendix),

$$
\begin{aligned}
& \nabla^{i} \nabla_{i}^{E} h^{\prime}\left(k T_{0}^{0}\right)=-\frac{1}{2 c^{2}} \frac{g_{E}^{00}}{g_{E}^{11}} \frac{\partial g_{E}^{11}}{\partial t} \frac{\partial h^{\prime}\left(k T_{0}^{0}\right)}{\partial t} . \\
& \int_{0}^{R_{0}(t)} \nabla^{i} \nabla_{i}^{E} h^{\prime}\left(k T_{0}^{0}\right) r^{\prime 2} d r^{\prime}
\end{aligned}
$$

$$
\approx h^{\prime \prime}\left(k T_{0}^{0}\right)\left[\frac{\partial}{\partial t} \frac{M}{\left[R_{0}(t)\right]^{3}}\right]^{2} \alpha(t),
$$

where,

$$
\begin{aligned}
\alpha(t)= & \frac{3 k^{2} c^{2} R_{0}(t)}{256 \pi^{2}[\xi(t)]^{4}}\left\{\frac{3}{\xi(t) R_{0}(t)} \arcsin \left[\xi(t) R_{0}(t)\right]\right. \\
& \left.-\left(3+2\left[\xi(t) R_{0}(t)\right]^{2}\right) \sqrt{1-\left[\xi(t) R_{0}(t)\right]^{2}}\right\} \\
& \times\left(1-\left[\xi(t) R_{0}(t)\right]^{2}\right)^{-3 / 2},
\end{aligned}
$$

with

$\xi^{2}(t)=\frac{k M c^{2}}{4 \pi\left[R_{0}(t)\right]^{3}}$.

Substituting (37) into (32) - (35) we find a solution at a distant point from the body-gravitation source:

$$
\begin{aligned}
g_{00}(r, t)=1 & -\frac{k c^{2} M}{4 \pi r} \\
& +\frac{\lambda}{2 r} \int_{0}^{r}\left[h\left(k T_{0}^{0}\right)+k T_{0}^{0} h^{\prime}\left(k T_{0}^{0}\right)\right] r^{\prime 2} d r^{\prime} \\
& +\frac{\lambda h^{\prime \prime}\left(k T_{0}^{0}\right)}{r}\left[\frac{\partial}{\partial t} \frac{M}{\left[R_{0}(t)\right]^{3}}\right]^{2} \alpha(t), \\
g_{11}(r, t)= & -\left\{1-\frac{k c^{2} M}{4 \pi r}\right. \\
& +\frac{\lambda}{2 r} \int_{0}^{r}\left[h\left(k T_{0}^{0}\right)+k T_{0}^{0} h^{\prime}\left(k T_{0}^{0}\right)\right] r^{\prime 2} d r^{\prime} \\
& \left.+\frac{\lambda h^{\prime \prime}\left(k T_{0}^{0}\right)}{r}\left[\frac{\partial}{\partial t} \frac{M}{\left[R_{0}(t)\right]^{3}}\right]^{2} \alpha(t)\right\}^{-1}, \\
g_{22}= & -r^{2}, \\
g_{33}= & -r^{2} \sin ^{2} \theta,
\end{aligned}
$$

here (26) is used for both inside and outside the integral, and $h^{\prime \prime}\left(k T_{0}^{0}\right)=\frac{\partial^{2} h\left(k T_{0}^{0}\right)}{\partial\left(k T_{0}^{0}\right)^{2}}$. Note that though $T_{0}^{0}$ depends on time $t$ only, one should be careful when bring $h\left(k T_{0}^{0}\right)+$ $k T_{0}^{0} h^{\prime}\left(k T_{0}^{0}\right)$ out of the integral. If $h\left(k T_{0}^{0}\right)+k T_{0}^{0} h^{\prime}\left(k T_{0}^{0}\right)=$ 0 in vacuum, the integral is performed inside the bodygravitation source, but there are also cases when $h\left(k T_{0}^{0}\right)+$ $k T_{0}^{0} h^{\prime}\left(k T_{0}^{0}\right)$ is not zero in vacuum (see below). Now, in the next subsection, applying the latest formulas, we consider some special cases.

\subsubsection{The case $f(R)=R-2 \lambda($ model I)}

In this case we have $h(R)=-2$ leading to $h\left(k T^{0}{ }_{0}\right)=-2$, $h^{\prime}\left(k T_{0}^{0}\right)=0$ and the formulas from (40) to (43) can be calculated easily as 


$$
\begin{aligned}
g_{00}(r, t) & =1-\frac{k c^{2} M}{4 \pi r}-\frac{\lambda r^{2}}{3}, \\
g_{11}(r, t) & =\frac{-1}{1-\frac{k c^{2} M}{4 \pi r}-\frac{\lambda r^{2}}{3}}, \\
g_{22} & =-r^{2}, \\
g_{33} & =-r^{2} \sin ^{2} \theta .
\end{aligned}
$$

It is exactly the solution of the modified Einstein equation with a cosmological constant $\lambda$.

\subsubsection{The case $f(R)=R+\lambda R^{b}, b>0$ (model II)}

Thus, $h(R)=R^{b}$ and $h^{\prime}(R)=b R^{b-1}, h^{\prime \prime}(R)=b(b-$ 1) $R^{b-2}$, the formulas (40)-(43) become

$$
\begin{aligned}
g_{00}=1 & -\frac{k c^{2} M}{4 \pi r}+\frac{\lambda(b+1) k^{b}}{2 r} \int_{0}^{R_{o}(t)}\left[T_{0}^{0}\right]^{b} r^{\prime 2} d r^{\prime} \\
& +\frac{\lambda}{r} b(b-1) k^{b-2}\left(T_{0}^{0}\right)^{b-2}\left[\frac{\partial}{\partial t} \frac{M}{\left[R_{o}(t)\right]^{3}}\right]^{2} \alpha(t), \\
g_{11}= & -\left\{1-\frac{k c^{2} M}{4 \pi r}+\frac{\lambda(b+1) k^{b}}{2 r} \int_{0}^{R_{o}(t)}\left[T_{0}^{0}\right]^{b} r^{\prime 2} d r^{\prime}\right. \\
& \left.+\frac{\lambda}{r} b(b-1) k^{b-2}\left(T_{0}^{0}\right)^{b-2}\left[\frac{\partial}{\partial t} \frac{M}{\left[R_{o}(t)\right]^{3}}\right]^{2} \alpha(t)\right\}^{-1},
\end{aligned}
$$

$g_{22}=-r^{2}$,

$g_{33}=-r^{2} \sin ^{2} \theta$.

Further, applying (26) we have

$$
\begin{aligned}
& g_{00}(r, t)=1-\frac{k c^{2}\left[M-\lambda M_{1}(t)-\lambda M_{2}(t)\right]}{4 \pi r}, \\
& g_{11}(r, t)=\frac{-1}{1-\frac{k c^{2}\left[M-\lambda M_{1}(t)-\lambda M_{2}(t)\right]}{4 \pi r}}, \\
& g_{22}=-r^{2}, \\
& g_{33}=-r^{2} \sin ^{2} \theta .
\end{aligned}
$$

Here

$$
\begin{aligned}
& M_{1}(t)=\frac{4 \pi}{k c^{2}} \frac{(b+1) c^{2 b}(k M)^{b}}{3^{1-b} 2^{2 b+1} \pi^{b}\left[R_{o}(t)\right]^{3 b-3}} \\
& M_{2}(t)=\frac{4 \pi}{k c^{2}} \frac{b(b-1) c^{2 b-4}(3 k M)^{b-2}\left[\frac{\partial}{\partial t} \frac{M}{\left[R_{o}(t)\right]^{3}}\right]^{2} \alpha(t)}{(4 \pi)^{b-2}\left[R_{o}(t)\right]^{3 b-6}} .
\end{aligned}
$$

\subsubsection{The case $f(R)=R^{1+\varepsilon}($ model III $)$}

Here $\varepsilon$ is an infinitesimally small number. In this case $\lambda h(R)=R^{1+\varepsilon}-R$ and $\lambda h^{\prime}(R)=(1+\varepsilon) R^{\varepsilon}-1, \lambda h^{\prime \prime}(R)=$ $\varepsilon(\varepsilon+1) R^{\varepsilon-1}$. Similarly, we obtain the corresponding metric tensor

$g_{00}(r, t)=1-\frac{k c^{2}\left[M-\lambda M_{1}(t)-\lambda M_{2}(t)\right]}{4 \pi r}$,

$g_{11}(r, t)=\frac{-1}{1-\frac{k c^{2}\left[M-\lambda M_{1}(t)-\lambda M_{2}(t)\right]}{4 \pi r}}$,

$g_{22}=-r^{2}$,

$g_{33}=-r^{2} \sin ^{2} \theta$

with

$\lambda M_{1}(t)=-M+\frac{4 \pi}{k c^{2}} \frac{(\varepsilon+2) 6^{\varepsilon}\left(k c^{2} M\right)^{\varepsilon+1}}{(8 \pi)^{(\varepsilon+1)}\left[R_{o}(t)\right]^{3 \varepsilon}}$,

$\lambda M_{2}(t)=\frac{4 \pi}{k c^{2}} \frac{\varepsilon(\varepsilon+1)\left(3 k c^{2} M\right)^{\varepsilon-1}\left[\frac{\partial}{\partial t} \frac{M}{\left[R_{o}(t)\right]^{3}}\right]^{2} \alpha(t)}{(4 \pi)^{\varepsilon-1}\left[R_{o}(t)\right]^{3 \varepsilon-3}}$.

We see, for example, in (52) or (58), that the metric in an $f(R)$-gravity is different from the one in Einstein's GR by last two terms. If the body-gravitation source shrinks or expands (it means that its radius depends on time), the metric would depend on time, unlike the Einstein equation giving no such a effect.

\subsection{General perturbative solution}

In the previous subsection, vacuum solutions have been found for an arbitrary and some more special $f(R)$, now we will look for a general solution everywhere, not only in vacuum. Inside matter we do not have $u(r, t)=-v(r, t)$, thus we will solve this problem in the following way: Doing the same calculations for obtaining formula (22) we get

$\square^{E} h^{\prime}(k T)-\nabla^{1} \nabla_{1}^{E} h^{\prime}(k T)=\beta(r, t)$,

where,

$$
\begin{aligned}
\beta(r . t)= & \frac{g_{E}^{00}}{c^{2}} \frac{\partial^{2} h^{\prime}(k T)}{\partial t^{2}}+\frac{1}{2 c^{2}} \frac{\partial g_{E}^{00}}{\partial t} \frac{\partial h^{\prime}(k T)}{\partial t} \\
& +g_{E}^{11}\left(\frac{2}{r}-\frac{1}{2 g_{E}^{00}} \frac{\partial g_{E}^{00}}{\partial r}\right) \frac{\partial h^{\prime}(k T)}{\partial r} .
\end{aligned}
$$

The index $E$ means the metric tensor taken within the Einstein theory (see the appendix for its values inside or outside the body-gravitation source). Substituting (64) into (9) we obtain the equation 


$$
\begin{aligned}
R_{1}^{1}-\frac{1}{2} R= & -k T_{1}^{1}+\frac{\lambda}{2} h(k T)+\lambda k\left(T_{1}^{1}-\frac{1}{2} T\right) h^{\prime}(k T) \\
& +\lambda \beta(r, t)
\end{aligned}
$$

which with using (30) leads to

$$
\begin{aligned}
& e^{-v(r, t)}\left[\frac{u^{\prime}(r, t)}{r}+\frac{1}{r^{2}}\right]-\frac{1}{r^{2}}=-k T_{1}^{1}+\frac{\lambda}{2} h(k T) \\
& +\lambda k\left(T_{1}^{1}-\frac{1}{2} T\right) h^{\prime}(k T)+\lambda \beta(r, t),
\end{aligned}
$$

or

$$
\begin{aligned}
u^{\prime}(r, t)= & r\left\{e ^ { v ( r , t ) } \left[\frac{1}{r^{2}}-k T_{1}^{1}+\frac{\lambda}{2} h(k T)\right.\right. \\
& \left.\left.+\lambda k\left(T_{1}^{1}-\frac{1}{2} T\right) h^{\prime}(k T)+\lambda \beta(r, t)\right]-\frac{1}{r^{2}}\right\} .
\end{aligned}
$$

Since $e^{v(r, t)}=-g_{11}(r, t)$, we can re-write the latter equation as

$$
\begin{aligned}
u^{\prime}(r, t)= & r g_{11}(r, t)\left[-\frac{1}{r^{2}}+k T_{1}^{1}-\frac{\lambda}{2} h(k T)\right. \\
& \left.-\lambda k\left(T_{1}^{1}+\frac{1}{2} T\right) h^{\prime}(k T)-\lambda \beta(r, t)\right]-\frac{1}{r} .
\end{aligned}
$$

Doing integration of the above equation and noticing that $g_{00}(r, t) \rightarrow 1$ as $r \rightarrow \infty$, we have

$$
\begin{aligned}
u(r, t)= & \int_{\infty}^{r}\left\{r ^ { \prime } g _ { 1 1 } ( r ^ { \prime } , t ) \left[-\frac{1}{r^{\prime 2}}+k T_{1}^{1}-\frac{\lambda}{2} h(k T)\right.\right. \\
& \left.\left.-\lambda k\left(T_{1}^{1}+\frac{1}{2} T\right) h^{\prime}(k T)-\lambda \beta\left(r^{\prime}, t\right)\right]-\frac{1}{r^{\prime}}\right\} d r^{\prime} .
\end{aligned}
$$

Thus, from (11), (18) and (70) the metric gets the form

$$
\begin{aligned}
g_{11}(r, t)= & -\left\{1-\frac{1}{r} \int_{0}^{r}\left[k T_{0}^{0}-\frac{\lambda}{2} h(k T)\right.\right. \\
& -\lambda k\left(T_{0}^{0}-\frac{T}{2}\right) h^{\prime}(k T) \\
& \left.\left.-\lambda \nabla^{i} \nabla_{i}^{E} h^{\prime}(k T)\right] r^{\prime 2} d r^{\prime}\right\}^{-1} \\
g_{22}= & -r^{2} \\
g_{33}= & -r^{2} \sin ^{2} \theta
\end{aligned}
$$$$
g_{00}(r, t)=\exp \int_{\infty}^{r}\left\{r ^ { \prime } g _ { 1 1 } ( r ^ { \prime } , t ) \left[-\frac{1}{r^{\prime 2}}+k T_{1}^{1}-\frac{\lambda}{2} h(k T)\right.\right.
$$$$
\left.\left.-\lambda k\left(T_{1}^{1}+\frac{1}{2} T\right) h^{\prime}(k T)-\lambda \beta\left(r^{\prime}, t\right)\right]-\frac{1}{r^{\prime}}\right\} d r^{\prime},
$$

where $\nabla^{i} \nabla_{i}^{E} h^{\prime}(k T)$ and $\beta\left(r^{\prime}, t\right)$ are given by (22) and (65), respectively.

\section{Motion in a central field}

In this section we will apply the obtained solutions to a motion in a central field, for example, a planetary motion around an isotropic star (which can be a normal star, neutron star, black hole, or other body-gravitational sources). This central field is not necessarily static, the radius of the star can expand or shrink during the time. Here we only take the models which satisfy $h(0)=0$, meaning that $h\left(k T_{0}^{0}\right)=0$, in vacuum [the models II and III, considered in Sects. 2.1.2 and 2.1.3, respectively, satisfy this, but the model I (in Subsect. 2.1.1) does not]. With these models, the integrations in (40)-(43) done only within the radius $R_{0}(t)$ of the star, lead to the solution

$g_{00}(r, t)=1-\frac{k c^{2}\left[M-\lambda M_{1}(t)-\lambda M_{2}(t)\right]}{4 \pi r}$,

$g_{11}(r, t)=\frac{-1}{1-\frac{k c^{2}\left[M-\lambda M_{1}(t)-\lambda M_{2}(t)\right]}{4 \pi r}}$,

$g_{22}=-r^{2}$,

$g_{33}=-r^{2} \sin ^{2} \theta$,

where

$$
\begin{aligned}
& M_{1}(t)=\frac{2 \pi\left[R_{o}(t)\right]^{3}}{3 k c^{2}}\left[h\left(k T_{0}^{0}\right)+k T_{0}^{0} h^{\prime}\left(k T_{0}^{0}\right)\right], \\
& M_{2}(t)=\frac{4 \pi}{k c^{2}} h^{\prime \prime}\left(k T_{0}^{0}\right)\left[\frac{\partial}{\partial t} \frac{M}{\left[R_{o}(t)\right]^{3}}\right]^{2} \alpha(t),
\end{aligned}
$$

with $T_{0}^{0}$ calculated by (26). For the model II, $M_{1}(t)$ and $M_{2}(t)$ have the form

$$
\begin{aligned}
& M_{1}(t)=\frac{4 \pi}{k c^{2}} \frac{(b+1) c^{2 b}(k M)^{b}}{3^{1-b} 2^{2 b+1} \pi^{b}\left[R_{o}(t)\right]^{3 b-3}}, \\
& M_{2}(t)=\frac{4 \pi}{k c^{2}} \frac{b(b-1) c^{2 b-4}(3 k M)^{b-2}\left[\frac{\partial}{\partial t} \frac{M}{\left[R_{o}(t)\right]^{3}}\right]^{2} \alpha(t)}{(4 \pi)^{b-2}\left[R_{o}(t)\right]^{3 b-6}} .
\end{aligned}
$$

and for the model III they become

$$
\begin{aligned}
& \lambda M_{1}(t)=-M+\frac{4 \pi}{k c^{2}} \frac{(\varepsilon+2) 6^{\varepsilon}\left(k c^{2} M\right)^{\varepsilon+1}}{(8 \pi)^{(\varepsilon+1)}\left[R_{o}(t)\right]^{3 \varepsilon}} \\
& \lambda M_{2}(t)=\frac{4 \pi}{k c^{2}} \frac{\varepsilon(\varepsilon+1)\left(3 k c^{2} M\right)^{\varepsilon-1}\left[\frac{\partial}{\partial t} \frac{M}{\left[R_{o}(t)\right]^{3}}\right]^{2} \alpha(t)}{(4 \pi)^{\varepsilon-1}\left[R_{o}(t)\right]^{3 \varepsilon-3}} .
\end{aligned}
$$


Setting

$M_{f}(t)=M-\lambda M_{1}(t)-\lambda M_{2}(t)$,

and noticing that $k=\frac{8 \pi G}{c^{4}}$, we have a Schwarzschild-type metric

$$
\begin{aligned}
d s^{2}= & {\left[1-\frac{2 G M_{f}(t)}{c^{2} r}\right] d x^{0^{2}}-\frac{d r^{2}}{1-\frac{2 G M_{f}(t)}{c^{2} r}} } \\
& -r^{2}\left(d \theta^{2}+\sin ^{2} \theta d \varphi^{2}\right) .
\end{aligned}
$$

Here, $M_{f}$ can be treated as an effective mass in an $f(R)$ gravity, which, then, looks like the GR in a central field of a source with a non-static mass $M_{f}$. In general, it is a function of time even when the mass $M$ is a constant. This may lead to interesting phenomena which can be discussed elsewhere later. Let us make a coordinate transformation changing $r$ as

$r \longrightarrow r\left[1+\frac{G M_{f}(t)}{2 c^{2} r}\right]^{2}$

but keeping other coordinates unchanged $(t \longrightarrow t, \theta \longrightarrow$ $\theta, \varphi \longrightarrow \varphi$ ). Subsituting (87) into (86) and neglecting the infinitesimal terms

$\frac{2 G}{c^{2}} \frac{\partial M_{f}(t)}{\partial t} \frac{\left[1+\frac{G M_{f}(t)}{2 c^{2} r}\right]^{4}}{1-\frac{G M_{f}(t)}{2 c^{2} r}} d t d r$,

and

$\frac{G^{2}}{c^{6}}\left(\frac{\partial M_{f}(t)}{\partial t}\right)^{2} \frac{\left[1+\frac{G M_{f}(t)}{2 c^{2} r}\right]^{4}}{\left[1-\frac{G M_{f}(t)}{2 c^{2} r}\right]^{2}} d x^{0^{2}}$,

very small compared with

$\frac{\left[1-\frac{G M_{f}(t)}{2 c^{2} r}\right]^{2}}{\left[1+\frac{G M_{f}(t)}{2 c^{2} r}\right]^{2}} d x 0^{0^{2}}$,

we get

$$
\begin{aligned}
d s^{2}= & \frac{\left[1-\frac{G M_{f}(t)}{2 c^{2} r}\right]^{2}}{\left[1+\frac{G M_{f}(t)}{2 c^{2} r}\right]^{2}} d x^{0^{2}}-\left[1+\frac{G M_{f}(t)}{2 c^{2} r}\right]^{4} \\
& \times\left(d r^{2}+r^{2} d \theta^{2}+r^{2} \sin ^{2} \theta d \varphi^{2}\right) .
\end{aligned}
$$

Since $d r^{2}+r^{2} d \theta^{2}+r^{2} \sin ^{2} \theta d \varphi^{2}=d x^{2}+d y^{2}+d z^{2}$, we have $g_{x x}=g_{y y}=g_{z z}$. It means that the spacial coordinates $x, y, z$ play the same role in the isotropic frame.
From the special relativity, we know the Hamilton-Jacobi equation of a free particle in a flat space-time,

$g^{\mu \nu} \frac{\partial S}{\partial x^{\mu}} \frac{\partial S}{\partial x^{\nu}}=m^{2} c^{2}$,

where $m$ and $S$ are its mass and action, respectively $[2,26]$. Since $S$ is a scalar, the equation (89) is still valid for the general relativity, where the flat space-time is replaced by a curved space-time. In the metric (88), the equation (89) has the form

$$
\begin{aligned}
& {\left[\frac{1+\frac{G M_{f}(t)}{2 c^{2} r}}{1-\frac{G M_{f}(t)}{2 c^{2} r}}\right]^{2}\left(\frac{\partial S}{\partial x^{0}}\right)^{2}-\frac{1}{\left[1+\frac{G M_{f}(t)}{2 c^{2} r}\right]^{4}}\left[\left(\frac{\partial S}{\partial r}\right)^{2}\right.} \\
& \left.+\frac{1}{r^{2}}\left(\frac{\partial S}{\partial \theta}\right)^{2}+\frac{1}{r^{2} \sin ^{2} \theta}\left(\frac{\partial S}{\partial \varphi}\right)^{2}\right]=m^{2} c^{2} .
\end{aligned}
$$

Let us apply this equation to a planet's motion around an isotropic star producing a central gravitational field. Since the planet moves in a fixed plane passing the star's center taken for the origin of the coordinate frame, we can choose the orientation of the coordinate frame so that the planet's orbital plane is horizontal, that is, we always have $\theta=\frac{\pi}{2}$ and, thus,

$$
\begin{aligned}
& {\left[\frac{1+\frac{G M_{f}(t)}{2 c^{2} r}}{1-\frac{G M_{f}(t)}{2 c^{2} r}}\right]^{2}\left(\frac{\partial S}{\partial x^{0}}\right)^{2}-\frac{1}{\left[1+\frac{G M_{f}(t)}{2 c^{2} r}\right]^{4}}\left[\left(\frac{\partial S}{\partial r}\right)^{2}\right.} \\
& \left.+\frac{1}{r^{2}}\left(\frac{\partial S}{\partial \varphi}\right)^{2}\right]=m^{2} c^{2} .
\end{aligned}
$$

\section{Because}

$\frac{\partial S}{\partial t}=-H(t)$

where $H(t)$ is the Hamiltonian, it follows that

$$
\begin{aligned}
& {\left[\frac{1+\frac{G M_{f}(t)}{2 c^{2} r}}{1-\frac{G M_{f}(t)}{2 c^{2} r}}\right]^{2}\left[\frac{H(t)}{c}\right]^{2}-\frac{1}{\left[1+\frac{G M_{f}(t)}{2 c^{2} r}\right]^{4}}\left[\left(\frac{\partial S}{\partial r}\right)^{2}\right.} \\
& \left.+\frac{1}{r^{2}}\left(\frac{\partial S}{\partial \varphi}\right)^{2}\right]=m^{2} c^{2} .
\end{aligned}
$$

In a central field which may not be static, the Hamiltonian may not be conserved but the angular momentum is always conserved. Following [26], we write

$$
\frac{\partial S}{\partial \varphi}=p_{\varphi}=\mu
$$


where $\mu$ is the conserved value of the angular momentum $p_{\varphi}$, or

$S=\mu \varphi+s(r, t)$,

where $s(r, t)$ is a function of $r$ and $t$ only. Taking (95) into account, Eq. (93) becomes

$\left[\frac{1+\frac{G M_{f}(t)}{2 c^{2} r}}{1-\frac{G M_{f}(t)}{2 c^{2} r}}\right]^{2}\left[\frac{H(t)}{c}\right]^{2}-\frac{\left[\frac{\partial s(r, t)}{\partial r}\right]^{2}+\frac{\mu^{2}}{r^{2}}}{\left[1+\frac{G M_{f}(t)}{2 c^{2} r}\right]^{4}}=m^{2} c^{2}$.

Solving the latter equation for $s(r, t)$ we get

$$
\begin{aligned}
& s(r, t)= \\
& \int\left\{\left[1+\frac{G M_{f}(t)}{2 c^{2} r}\right]^{4}\left(\left[\frac{1+\frac{G M_{f}(t)}{2 c^{2} r}}{1-\frac{G M_{f}(t)}{2 c^{2} r}}\right]^{2}\left[\frac{H(t)}{c}\right]^{2}-m^{2} c^{2}\right)\right. \\
& \left.-\frac{\mu^{2}}{r^{2}}\right\}^{1 / 2} d r .
\end{aligned}
$$

Note that in the above integral the coordinates $r$ and $t$ are treated as independent variables. As $\frac{\partial S}{\partial \mu}$ is also a constant of motion $[2,26]$,

$$
\frac{\partial S}{\partial \mu}=\text { const. }
$$

it means

$\varphi=-\frac{\partial s(r, t)}{\partial \mu}+$ const..

Combining (97) with (99), we find

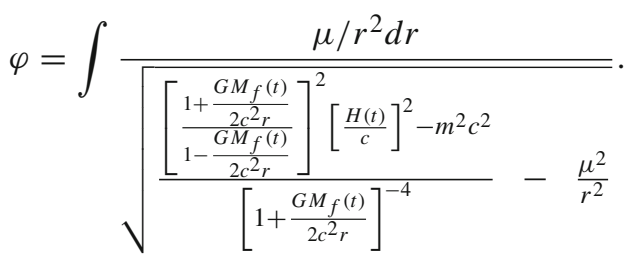

\subsection{Motion of a planet in a central field of a star}

Let us consider the motion of a planet around an isotropic star. If we write the Hamiltonian in the form

$H(t)=E(t)+m c^{2}$,

[where $E(t)$ has the meaning of both kinetic energy and potential energy of the planet in the gravitational field], then (100) is rewritten as

$$
\varphi=\int \frac{\mu / r^{2} d r}{\sqrt{\frac{\left[\frac{1+\frac{G M_{f}(t)}{2 c^{2} r}}{1-\frac{G M_{f}(t)}{2 c^{2} r}}\right]^{2}\left[\frac{E^{2}(t)+2 m c^{2} E(t)+m^{2} c^{4}}{c^{2}}\right]-m^{2} c^{2}}{\left[1+\frac{G M_{f}(t)}{2 c^{2} r}\right]^{-4}}-\frac{\mu^{2}}{r^{2}}}} .
$$

On the other hand, if we consider the planet's motion to be relatively slow (compared with the light), $\frac{v^{2}}{c^{2}} \ll 1$, where, $v$ is the planet's speed, that is, $E^{2} \ll\left|2 m c^{2} E\right|$, we have

$$
\varphi=\int \frac{\mu / r^{2} d r}{\sqrt{\frac{\left[\frac{1+\frac{G M_{f}(t)}{2 c^{2} r}}{1-\frac{G M_{f}(t)}{2 c^{2} r}}\right]^{2}\left[2 m E(t)+m^{2} c^{2}\right]-m^{2} c^{2}}{\left[1+\frac{G M_{f}(t)}{2 c^{2} r}\right]^{-4}}-\frac{\mu^{2}}{r^{2}}}},
$$

or

$$
\begin{aligned}
& \varphi=\int d r \\
& \sqrt{\frac{2 m E(t)\left[\frac{1+\frac{G M_{f}(t)}{2 c^{2} r}}{1-\frac{G M_{f}(t)}{2 c^{2} r}}\right]^{2}}{\left[\frac{G r^{2}}{\left[1+\frac{G M_{f}(t)}{2 c^{2} r}\right]^{-4}}+\frac{2 m^{2} G M_{f}(t)}{r} \frac{\left[1+\frac{G M_{f}(t)}{2 c^{2} r}\right]^{4}}{\left[1-\frac{G M_{f}(t)}{2 c^{2} r}\right]^{2}}-\frac{\mu^{2}}{r^{2}}\right.}} .
\end{aligned}
$$

At the first order approximation

$$
\left[\frac{1+\frac{G M_{f}(t)}{2 c^{2} r}}{1-\frac{G M_{f}(t)}{2 c^{2} r}}\right]^{2} \cong\left[1+\frac{G M_{f}(t)}{2 c^{2} r}\right]^{4} \cong 1+\frac{2 G M_{f}(t)}{c^{2} r},
$$

the equation (104) takes the form

$$
\varphi=\int \frac{\mu / r^{2} d r}{\sqrt{\frac{2 m E(t)}{\left[1+\frac{4 G M_{f}(t)}{c^{2} r}\right]^{-1}}+\frac{\frac{2 m^{2} G M_{f}(t)}{r}}{\left[1+\frac{3 G M_{f}(t)}{c^{2} r}\right]^{-1}}-\frac{\mu^{2}}{r^{2}}}},
$$

that is,

$$
\varphi=\int d r
$$

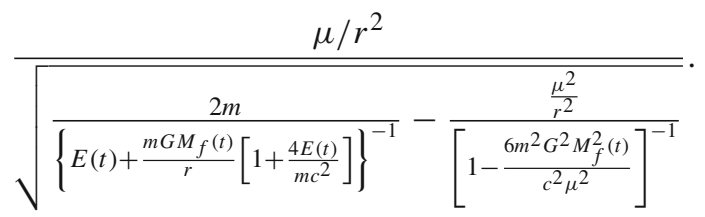


Using the notation

$$
\begin{aligned}
& \beta(t)=m G M_{f}(t)\left[1+\frac{4 E(t)}{m c^{2}}\right], \\
& l^{2}(t)=\mu^{2}\left[1-\frac{6 m^{2} G^{2} M_{f}^{2}(t)}{c^{2} \mu^{2}}\right],
\end{aligned}
$$

we obtain the formula

$$
\varphi=\frac{1}{\sqrt{1-\frac{6 m^{2} G^{2} M_{f}^{2}(t)}{c^{2} \mu^{2}}}} \int \frac{l(t) / r^{2}}{\sqrt{2 m\left[E(t)+\frac{\beta(t)}{r}\right]-\frac{l^{2}(t)}{r^{2}}}} d r
$$

which after doing integration becomes

$\varphi=\frac{1}{\sqrt{1-\frac{6 m^{2} G^{2} M_{f}^{2}(t)}{c^{2} \mu^{2}}}} \arccos \frac{\frac{l(t)}{r}-\frac{m \beta(t)}{l(t)}}{\sqrt{2 m E(t)+\frac{m^{2} \beta^{2}(t)}{l^{2}(t)}}}+C$,

where $C$ is a constant. Rotating the coordinate frame so that $C=0$,

$\varphi=\frac{1}{\sqrt{1-\frac{6 m^{2} G^{2} M_{f}^{2}(t)}{c^{2} \mu^{2}}}} \arccos \frac{\frac{l(t)}{r}-\frac{m \beta(t)}{l(t)}}{\sqrt{2 m E(t)+\frac{m^{2} \beta^{2}(t)}{l^{2}(t)}}}$,

we get

$\frac{l^{2}(t)}{m \beta(t) r}=$

$1+\sqrt{1+\frac{2 E(t) l^{2}(t)}{m \beta^{2}(t)}} \cos \left(\sqrt{1-\frac{6 m^{2} G^{2} M_{f}^{2}(t)}{c^{2} \mu^{2}}} \varphi\right)$.

This is the equation of motion of a planet in a central field of a star. We notice that $t=0$ is chosen arbitrarily after having "gravitational interaction" passing the planet. The Eqs. (110) or (112) or (113) is a general equation of a planetary motion in a central field of a star.

Now we consider a planet moving in a nearly-elliptic orbit. From (113) we can see that the lengths of the major axis and the minor axis of the near-elliptic orbit change if the central filed is not static (note that the central field is not static even when the total mass $M$ of the star is unchanged, if the star expands or shrinks keeping its isotropic form). This is an effect which cannot occur in the Einstein theory [when the radius of the star expands or shrinks, the metric in the vacuum in the Einstein theory does not depend on time]. The extremums (apsides) $r_{e}$ of $r$, which are its minimal value (periastron/perihelion) $r_{p}$ or maximal value (apastron/aphelion) $r_{a}$, can be calculated as follows: First, we notice that the argument of an arccos fuction varies within the interval $[-1,1]$, hence from $(112)$ we have the condition

$-1 \leqslant \frac{\frac{l(t)}{r}-\frac{m \beta(t)}{l(t)}}{\sqrt{2 m E(t)+\frac{m^{2} \beta^{2}(t)}{l^{2}(t)}}} \leqslant 1$.

The extremums $r_{e}$ are found at the two edges of this interval,

$\frac{\frac{l\left(t_{e}\right)}{r_{e}}-\frac{m \beta\left(t_{e}\right)}{l\left(t_{e}\right)}}{\sqrt{2 m E\left(t_{e}\right)+\frac{m^{2} \beta^{2}\left(t_{e}\right)}{l^{2}\left(t_{e}\right)}}}= \pm 1$,

where, $t_{e}$ is the time value corresponding to $r_{e}$. Hence,

$r_{e}=\frac{l^{2}\left(t_{e}\right)}{m \beta\left(t_{e}\right) \pm \sqrt{m^{2} \beta^{2}\left(t_{e}\right)+2 m E\left(t_{e}\right) l^{2}\left(t_{e}\right)}}$

or, more precisely,

$r_{p}=\frac{l^{2}\left(t_{p}\right)}{m \beta\left(t_{p}\right)+\sqrt{m^{2} \beta^{2}\left(t_{p}\right)+2 m E\left(t_{n}\right) l^{2}\left(t_{p}\right)}}$,

$r_{a}=\frac{l^{2}\left(t_{a}\right)}{m \beta\left(t_{a}\right)-\sqrt{m^{2} \beta^{2}\left(t_{a}\right)+2 m E\left(t_{a}\right) l^{2}\left(t_{a}\right)}}$.

From (112), we have

$\varphi_{e}=\frac{1}{\sqrt{1-\frac{6 m^{2} G^{2} M_{f}^{2}\left(t_{e}\right)}{c^{2} \mu^{2}}}} \arccos \frac{\frac{l\left(t_{e}\right)}{r_{e}}-\frac{m \beta\left(t_{e}\right)}{l\left(t_{e}\right)}}{\sqrt{2 m E\left(t_{e}\right)+\frac{m^{2} \beta^{2}\left(t_{e}\right)}{l^{2}\left(t_{e}\right)}}}$,

and with (116) substituted into (119) we obtain

$\varphi_{e}=\frac{1}{\sqrt{1-\frac{6 m^{2} G^{2} M_{f}^{2}\left(t_{e}\right)}{c^{2} \mu^{2}}}} \arccos ( \pm 1)$,

for $e$ being $p$ or $a$ (but not both simultaneously). It follows that

$\varphi_{e}(k)=\frac{k \pi}{\sqrt{1-\frac{6 m^{2} G^{2} M_{f}^{2}\left(t_{k}\right)}{c^{2} \mu^{2}}}} \quad(k \in \mathbb{Z})$,

with $\varphi_{e}(k)$ being the set of all the values of the angle $\varphi$ at which $r$ gets an extremum value $r_{e}$, where, $k$ is even for $e=p$ and odd for $e=a$. Thus, the orbital precession is

$\Delta \varphi_{e}(k)=\varphi_{e}(k+1)-\varphi_{e}(k)=$ 
$\frac{2(k+1) \pi}{\sqrt{1-\frac{6 m^{2} G^{2} M_{f}^{2}\left(t_{k+1}\right)}{c^{2} \mu^{2}}}}-\frac{2 k \pi}{\sqrt{1-\frac{6 m^{2} G^{2} M_{f}^{2}\left(t_{k}\right)}{c^{2} \mu^{2}}}}(\bmod 2 \pi)$.

If $M_{f}\left(t_{k+1}\right) \cong M_{f}\left(t_{k}\right)$, Eq. (122) can be taken approximately as

$$
\begin{aligned}
\Delta \varphi_{e}(k) & \cong \frac{2 \pi}{\sqrt{1-\frac{6 m^{2} G^{2} M_{f}^{2}\left(t_{k}\right)}{c^{2} \mu^{2}}}}(\bmod 2 \pi) \\
& \cong 2 \pi\left[1+\frac{3 m^{2} G^{2} M_{f}^{2}\left(t_{k}\right)}{c^{2} \mu^{2}}\right](\bmod 2 \pi) .
\end{aligned}
$$

Thus, the orbital precession becomes

$\Delta \varphi_{e}(k)=\frac{6 \pi m^{2} G^{2} M_{f}^{2}\left(t_{k}\right)}{c^{2} \mu^{2}}$.

Therefore, the correction to Einstein's precession is

$$
\begin{aligned}
& \delta \varphi_{e}(k)= \\
& \frac{6 \pi m^{2} G^{2}\left\{\lambda^{2}\left[M_{1}\left(t_{k}\right)+M_{2}\left(t_{k}\right)\right]^{2}-2 \lambda M\left[M_{1}\left(t_{k}\right)+M_{2}\left(t_{k}\right)\right]\right\}}{c^{2} \mu^{2}},
\end{aligned}
$$

or

$\delta \varphi_{e}(k) \cong \frac{-12 \pi m^{2} G^{2} \lambda M\left[M_{1}\left(t_{k}\right)+M_{2}\left(t_{k}\right)\right]}{c^{2} \mu^{2}}$

when the second order approximation is neglected. If the central field is static, $\Delta \varphi_{e}$ (thus, $\delta \varphi_{e}$ ) is a constant (see Fig. 1 which is only illustrative), but when the central field is not static (for example when the radius of the star expands or shrinks keeping the star's isotropy) $\Delta \varphi_{e}$ (thus, $\delta \varphi_{e}$ ) is no longer a constant, and there will be new effects compared with Eisntein's theory: there is not only a corrected orbital precession given by (124) and (126), but, as shown in (117) and (118), the axes of the orbit also varies with time (see an illustration in Fig. 2).

\subsection{Light's propagation in the central field of a star}

Since $m=0$ in this case, Eq. (100) is reduced to

$$
\varphi=\int \frac{\mu / r^{2}}{\sqrt{\left[1+\frac{G M_{f}(t)}{2 c^{2} r}\right]^{4}\left[\frac{1+\frac{G M_{f}(t)}{2 c^{2} r}}{1-\frac{G M_{f}(t)}{2 c^{2} r}}\right]^{2}\left[\frac{H(t)}{c}\right]^{2}-\frac{\mu^{2}}{r^{2}}}} d r .
$$

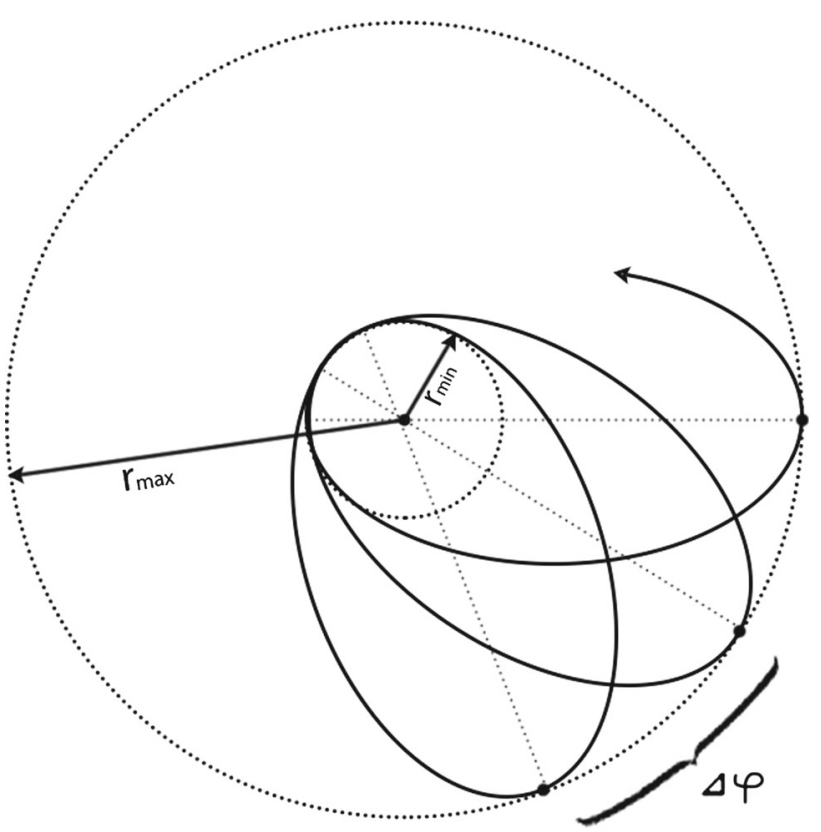

Fig. 1 In a static central field, both $r_{e}$ and $\Delta \varphi$ remain unchanged over time as in Einstein's theory but have a correction to the corresponding Einstein's values (illustration following [26])

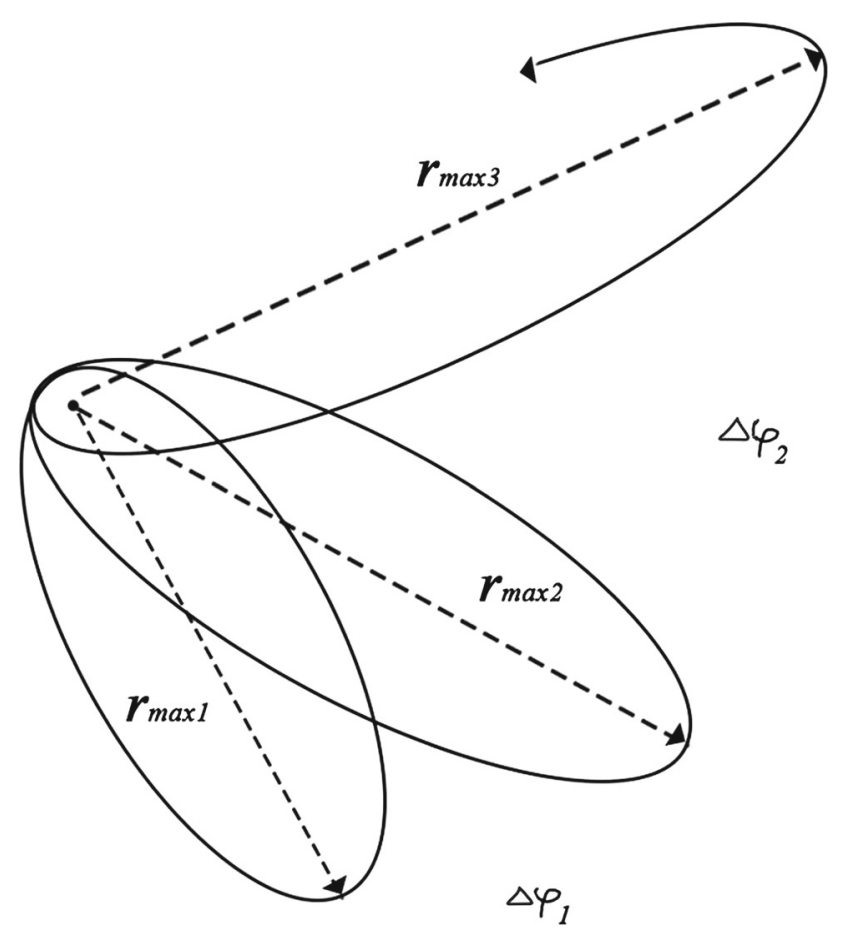

Fig. 2 In a non-static case, both $r_{e}$ and $\Delta \varphi$ change over time, unlike the corresponding Einstein's values remaning always unchanged in a central field (from a source with a constant mass)

Then, using (105), we obtain

$\varphi=\int \frac{\mu / r^{2}}{\sqrt{\left[1+\frac{4 G M_{f}(t)}{c^{2} r}\right]\left[\frac{H(t)}{c}\right]^{2}-\frac{\mu^{2}}{r^{2}}}} d r$. 
At the first order of approximation, it follows that

$\varphi=\int \frac{\mu / r^{2}}{\sqrt{\frac{H^{2}(t)}{c^{2}}-\left[\frac{2 G M_{f}(t) H^{2}(t)}{c^{4} \mu}-\frac{\mu}{r}\right]^{2}}} d r$

or

$\varphi=\arccos \left[\frac{\mu c}{H(t) r}-\frac{2 G M_{f}(t) H(t)}{c^{3} \mu}\right]+C^{\prime}$,

where $C^{\prime}$ is a constant. If the reference frame is chosen so that $\varphi=0$ at the point (periastron/perihelion) where the light is closest to the origin of the frame (the gravitational source), we can take $C^{\prime}=0$. Thus, the equation of motion of light in a central field is

$\varphi=\arccos \left[\frac{\mu c}{H(t) r}-\frac{2 G M_{f}(t) H(t)}{c^{3} \mu}\right]$,

or

$\cos \varphi=\frac{\mu c}{H(t) r}-\frac{2 G M_{f}(t) H(t)}{c^{3} \mu}$.

At a far-distance (with $r \equiv r_{l}$ very large), this equation has the approximate form

$\cos \varphi_{l}=-\frac{2 G M_{f}\left(t_{l}\right) H\left(t_{l}\right)}{c^{3} \mu}$.

It means that $\varphi_{l}>\frac{\pi}{2}$, that is, the light's trajectory is deflected toward the gravitational source, and the deflection angle, denoted by $\phi$, is

$$
\begin{aligned}
\phi & =2\left(\varphi_{l}-\frac{\pi}{2}\right) \\
& =2 \varphi_{l}-\pi .
\end{aligned}
$$

This angle (135) can be calculated approximately as follows. Denoting the periastron distance by $D$ (a notation used in [2]). As $\varphi=0$ at $r=D$, from (132) we have

$\frac{\mu c}{H\left(t_{D}\right) D}-\frac{2 G M_{f}\left(t_{D}\right) H\left(t_{D}\right)}{c^{3} \mu}=1$,

it follows that

$\mu=\frac{H\left(t_{D}\right) D}{2 c}\left(1+\sqrt{1+\frac{8 G M_{f}\left(t_{D}\right)}{c^{2} D}}\right)$.

Neglecting again higher orders of approximation, we have

$\mu \cong \frac{H\left(t_{D}\right) D}{c}\left(1+\frac{2 G M_{f}\left(t_{D}\right)}{c^{2} D}\right)$, or

$\frac{1}{\mu} \cong \frac{c}{H\left(t_{D}\right) D}\left(1-\frac{2 G M_{f}\left(t_{D}\right)}{c^{2} D}\right)$

Substituting (139) into (133) we obtain

$\cos \varphi_{l}=-\frac{2 G M_{f}\left(t_{l}\right)}{c^{2} D} \frac{H\left(t_{l}\right)}{H\left(t_{D}\right)}+\frac{4 G^{2} M_{f}\left(t_{a}\right) M_{f}\left(t_{D}\right)}{c^{4} D^{2}} \frac{H\left(t_{l}\right)}{H\left(t_{D}\right)}$,

where, the second term is very small compared with the first term. Thus, we can take

$\cos \varphi_{l}=-\frac{2 G M_{f}\left(t_{l}\right)}{c^{2} D} \frac{H\left(t_{l}\right)}{H\left(t_{D}\right)}$

From (141) we see the RHS term is very small and $\varphi_{l}>\frac{\pi}{2}$, and we can set

$\varphi_{l}=\frac{\pi}{2}+\varepsilon$

with $\varepsilon$ being very small. Combining (141) with (142), we have

$\cos \left(\frac{\pi}{2}+\varepsilon\right)=-\sin \varepsilon \cong-\frac{2 G M_{f}\left(t_{l}\right)}{c^{2} D} \frac{H\left(t_{l}\right)}{H\left(t_{D}\right)}$,

or

$\varepsilon \cong \frac{2 G M_{f}\left(t_{l}\right)}{c^{2} D} \frac{H\left(t_{l}\right)}{H\left(t_{D}\right)}$,

and, thus,

$\varphi_{l}=\frac{\pi}{2}+\frac{2 G M_{f}\left(t_{l}\right)}{c^{2} D} \frac{H\left(t_{l}\right)}{H\left(t_{D}\right)}$.

From here, taking (135) into account, we have

$\phi=\frac{4 G M_{f}\left(t_{l}\right)}{c^{2} D} \frac{H\left(t_{l}\right)}{H\left(t_{D}\right)}$.

We can see that if the gravitational field is static $H\left(t_{l}\right)=$ $H\left(t_{D}\right)=H$ then $\phi=\frac{4 G M_{f}}{c^{2} D}$ with $M_{f}=M-\lambda M_{1}$ [see (85)], and this angle has a correction to Einstein's value

$\delta \phi=-\lambda \frac{4 G M_{1}}{c^{2} D}$.

In an $f(R)$ theory, the light deflection angle, like the orbital precession discussed above, in a static central gravitational field, has a constant correction to that in Einstein's theory, but when the gravitational field is not static the correction may not be a constant, but, in general, it depends on time. 


\section{Conclusions}

Einstein's general theory of relativity is a triumphant theory but, as mentioned above, it meets several open problems such as the accelerated expansion of the Universe (or dark energy), the cosmic inflation, an integration with quantum theory (quantum gravity), etc. The $f(R)$-theory of gravity was introduced to solve some of these problems. Then, the Einstein equation (2) is replaced by a, more complicated in general, Eq. (6). Usually, solving the latter is problematic and it must be done via an approximation method by imposing appropriate condition(s). As, physically, the $f(R)$-gravity is assumed to be a perturbative theory around Einstein's GR describing very well most of today observations, we have followed a perturbation approach to solving Eq. (6). However, even with this assumption, it is not always easy to solve Eq. (6) without imposing any further condition. One of the most often imposed conditions is the spherical symmetry being a good approximation in many cases. Therefore, in this article we try to perturbatively solve Eq. (6) in a central field. The corresponding general solution is given in (71)-(74), while the vacuum solution is given in (32)-(35). At a large distance from the gravitational source the solution (32)-(35) can be written in the form (40)-(43) with some particular cases also considered (see 2.1.1-2.1.3). These results, as discussed in Sect. 3, can be applied to investigating planetary and light's motions in a central field. In comparison with Einstein's theory, an orbital precession or a trajectory deflection now gets a correction which is a constant for a static central field and varies with time for a non-static central field even from a source of a constant mass, unlike the corresponding Einstein's value which does not change in the same circumstance. In general, a spherically symmetric vacuum solution of Eq. (6) is not stationary, while a spherically symmetric vacuum solution of the Einstein equation is always stationary. In other words, Birkhoff's theorem in the GR is not valid any more for a general $f(R)$-theory of gravity. This may have interesting consequences (for example, a spherically symmetric pulsating (or expanding or collapsing) object is not disabled to emit gravitational waves as in the GR) being a subject of our next investigation. Following the present method, we will also investigate cosmological equations and models corresponding to the $f(R)$-theory of gravity.

The results obtained above may give an indication for an experimental test of an $f(R)$-theory of gravity. This theory in the considered circumstance can be treated as an Einstein's GR with an effective mass $\left(M_{f}\right)$ which may vary with time even in the case keeping the original total mass $(M)$ constant. Let us make some estimation using a real data.

As seen above, a perturbative $f(R)$-theory can be considered as Einstein theory with an effective mass $M_{f}=$ $M-\lambda M_{1}-\lambda M_{2}$ replacing the original mass $M$ assumed here to be a constant. According to (80) $M_{2}=0$ for a static field, this effective mass becomes $M_{f}=M-\lambda M_{1}$. Thus, from (124) we have $\Delta \varphi=\frac{6 \pi G^{2} m^{2}\left(M-\lambda M_{1}\right)^{2}}{c^{2} \mu^{2}}$ or

$\lambda M_{1}=M-\sqrt{\frac{c^{2} \mu^{2}}{6 \pi G^{2} m^{2}} \Delta \varphi}$.

Putting $\frac{\mu^{2}}{G M m^{2}}=a\left(1-e^{2}\right)$ in the latter Eq. (148), where, $a$ is the length of a semi-major axis and $e$ is the eccentricity of an orbital ellipse [2], we get

$\lambda M_{1}=M-\sqrt{\frac{c^{2} M}{6 \pi G} a\left(1-e^{2}\right) \Delta \varphi}$.

Using a recent data for the Mercury's orbital precession [27]: $c=299792458 \mathrm{~m} / \mathrm{s} ; G=6.67259 \times 10^{-11} \mathrm{~kg}^{-1} \mathrm{~m}^{3} \mathrm{~s}^{-2}$; $\frac{2 G M}{c^{2}}=2.95325008 \times 10^{3} \mathrm{~m} ; a=5.7909175 \times 10^{10} \mathrm{~m}$; $e=0.20563069 ; \Delta \varphi_{o b s}=2 \pi(7.98734 \pm 0,00037) \times$ $10^{-8}$ radian/revolution, we can estimate the correction $\lambda M_{1}$ to the mass $M$ to be

$\lambda M_{1}=11.866507 \times 10^{24} \mathrm{~kg}$.

It means that the Sun's mass $M=1.988919 \times 10^{30} \mathrm{~kg}$ is effectively reduced by

$\frac{\lambda M_{1}}{M}=5.966309 \times 10^{-6}=0.0005966309 \%$.

It is quite small compared with the Sun's mass but the effect may be measurable (see below). All these results on $\lambda M_{1}$ are model-independent, i.e., for an arbitrary $f(R)$. To estimate $\lambda$ we need, however, a concrete $f(R)$.

According to a perturbation criterion $\lambda h(R) \ll R$ applied to (7) and taking (4) into account we have

$\lambda h(T) \ll \frac{8 \pi G}{c^{4}} T$,

or for $T \approx T_{0}^{0}$,

$\lambda h\left(T_{0}^{0}\right) \ll \frac{8 \pi G}{c^{4}} T_{0}^{0}$.

Inserting $T_{0}^{0}=\frac{M c^{2}}{\frac{4}{3} \pi\left[R_{0}\right]^{3}}$ (where $R_{0}$ and $M$ are the radius and the mass of the body-gravitational source, respectively) we get

$\lambda h \ll \frac{6 G M}{c^{2}\left[R_{0}\right]^{3}}$.

That means $\lambda h$ is very small,

$\lambda h \ll 26 \times 10^{-24}$, 
as expected, where the radius $R_{0}=6.957 \times 10^{8} \mathrm{~m}$ of the Sun (see wikipedia.org) is used. Note that the compatibility between (151) and (154) depends on the model chosen. For example, we choose the model

$f(R)=R+\lambda R^{b}$,

for $b=2$, that is, $h(R)=R^{2}$, and obeying (154) $\lambda$ must satisfy the condition

$\lambda \ll \frac{c^{2}\left[R_{0}\right]^{3}}{6 G M}$.

For the data given above, the latter inequality becomes

$\lambda \ll 0.380053 \times 10^{23}$.

From here, we can see also $\lambda h^{\prime}(R)=2 \lambda R \ll 1$. Using (150) with $M_{1}=78.4989635 \times 10^{6}$ calculated by (81) for the model (156) with $b=2$ we get the following value of $\lambda$

$\lambda=0.1511677 \times 10^{18}$,

which is compatible with (158), and, thus, consistent with the observed data. From here we have

$$
\begin{aligned}
\delta \varphi & =\Delta \varphi_{f(R)}-\Delta \varphi_{G R} \\
& =-0.1906 \pi \times 10^{-11} \mathrm{radian} / \text { revolution. }
\end{aligned}
$$

As $\lambda$ may not be always very small the choice of $h(R)$ to satisfiy the perturbation condition is very important. For the model (156) the smaller $b>0$ is chosen the smaller $\lambda$ is obtained. For example, if $b$ has a value of the order $10^{-11}$ the value of $\lambda$ would be at the order $10^{-29}$. One should note that $\lambda$ is not an observable quantity but it can be fixed, as in (159) for example, for a given model by using an observed data.

Above, we have applied our results to the case of a motion around a star such as our Sun for which there is a very good experimental/observed data for reference. In order to improve the potential of an experimental detection of an $f(R)$-gravity effect, we can consider stronger gravitational systems like that of Sgr A* at the center of our Galaxy and orbiting it stars. The role of the Mercury is played now by $\mathrm{S} 2$ going around the "sun" Sgr A* which has a mass of $M=4.31 \times 10^{6} M_{\odot}=$ $8.57 \times 10^{36} \mathrm{~kg}$ and a radius of $R_{0}=22 \times 10^{9} \mathrm{~m}$. With the latter data and the orbital information of S2 $(a=0.123$ $\operatorname{arcsec}=14.7 \times 10^{13} \mathrm{~m}$ and $e=0.88$ ) [28], we can find the deviation between the S2's orbital precessions calulated by the GR and the $f(R)$-theory as

$$
\begin{aligned}
\delta \varphi^{S 2} & =\Delta \varphi_{f(R)}^{S 2}-\Delta_{G R}^{S 2} \\
& =-0.94 \pi \times 10^{-6} \text { radian/revolution, }
\end{aligned}
$$

with

$\Delta \varphi_{G R}^{S 2}=1.15114 \pi \times 10^{-3} \mathrm{radian} /$ revolution,

calculated by the GR, and

$\Delta \varphi_{f(R)}^{S 2}=1.1502 \pi \times 10^{-3}$ radian $/$ revolution,

calculated for the model $f(R)=R+\lambda R^{2}$ by using $\lambda$ obtained in (159) from the Sun-Mercury system. This deviation is much bigger than that given in (160) and also bigger than the observed orbital precession $\Delta \varphi_{o b s}$ of the Mercury, thus, much easier to be measured (with the condition that difficulties of measurement by other reasons, if any, are excluded or resolved).

In general, the deviation between the two theories, the GR and the $f(R)$-gravity, is very small but it is measurable if one can invent a measurement technique sensitive as that of the LIGO which is sensitive to a relative length change of an order of around $10^{-20}$.

Acknowledgements This research is funded by the National Foundation for Science and Technology Development (NAFOSTED) of Vietnam under contract No. 103.01-2017.76.

Open Access This article is distributed under the terms of the Creative Commons Attribution 4.0 International License (http://creativecomm ons.org/licenses/by/4.0/), which permits unrestricted use, distribution, and reproduction in any medium, provided you give appropriate credit to the original author(s) and the source, provide a link to the Creative Commons license, and indicate if changes were made.

Funded by SCOAP ${ }^{3}$.

\section{Appendix A: Einstein-Schwarzchild metric inside a body- gravitational source}

Now we prove formula (37). From (71) and (72) in the Einstein limit (taking $\lambda h$ to be zero) we have

$$
\begin{aligned}
& g_{11}(r, t)=\frac{-1}{1-\frac{1}{r} \int_{0}^{r} k T_{0}^{0}\left(r^{\prime}, t\right) r^{\prime 2} d r^{\prime}}, \\
& g_{00}(r, t)= \\
& \exp \int_{\infty}^{r}\left\{r^{\prime} g_{11}\left(r^{\prime}, t\right)\left[-\frac{1}{r^{\prime 2}}+k T_{1}^{1}\left(r^{\prime}, t\right)\right]-\frac{1}{r^{\prime}}\right\} d r .
\end{aligned}
$$

Since $T_{v}^{\mu}=0$ outside the gravitational source [of radius $\left.R_{O}(t)\right]$ the latter integral becomes

$$
\begin{aligned}
& g_{00}(r, t)= \\
& \exp \int_{R_{o}(t)}^{r}\left\{r^{\prime} g_{11}\left(r^{\prime}, t\right)\left[-\frac{1}{r^{\prime 2}}+k T_{1}^{1}\left(r^{\prime}, t\right)\right]-\frac{1}{r^{\prime}}\right\} d r^{\prime} \\
& \times \exp \int_{\infty}^{R_{o}(t)} \frac{-g_{11}\left(r^{\prime}, t\right)-1}{r^{\prime}} d r^{\prime}
\end{aligned}
$$


On the other hand, outside the object $g_{11}=\frac{-1}{1-\frac{2 G M}{c^{2} r}}$, hence,

$g_{00}(r, t)=$

$\exp \int_{R_{o}(t)}^{r}\left\{r^{\prime} g_{11}\left(r^{\prime}, t\right)\left[-\frac{1}{r^{\prime 2}}+k T_{1}^{1}\left(r^{\prime}, t\right)\right]-\frac{1}{r^{\prime}}\right\} d r^{\prime}$

$\times\left[1-\frac{2 G M}{c^{2} R_{o}(t)}\right]$.

From (A.1) and (A.4), it follows

$$
\begin{aligned}
& g_{00}(r, t)=\left[1-\frac{2 G M}{c^{2} R_{o}(t)}\right] \\
& \times \exp \int_{R_{o}(t)}^{r} \frac{\frac{k}{r^{\prime 2}} \int_{0}^{r^{\prime}} r^{\prime \prime 2} T_{0}^{0}\left(r^{\prime \prime}, t\right) d r^{\prime \prime}-k r^{\prime} T_{1}^{1}\left(r^{\prime}, t\right)}{1-\frac{k}{r^{\prime}} \int_{0}^{r^{\prime}} r^{\prime \prime 2} T_{0}^{0}\left(r^{\prime \prime}, t\right) d r^{\prime \prime}} d r^{\prime},
\end{aligned}
$$

but, as in (36) we consider $T_{0}^{0}$ depending on time $t$ only (the density is uniform as the body-gravitation source is considered homogeneous), therefore,

$$
\begin{aligned}
& g_{00}(r, t)=\left[1-\frac{2 G M}{c^{2} R_{o}(t)}\right] \\
& \times \exp \int_{R_{o}(t)}^{r} \frac{\frac{k}{3} r^{\prime} T_{0}^{0}(t)-k r^{\prime} T_{1}^{1}\left(r^{\prime}, t\right)}{1-\frac{k}{3} r^{\prime 2} T_{0}^{0}(t)} d r^{\prime} .
\end{aligned}
$$

Treating $T_{1}^{1}$ very small compared with $T_{0}^{0}$, we obtain $g_{00}(r, t)=\left[1-\frac{2 G M}{c^{2} R_{o}(t)}\right] \exp \int_{R_{o}(t)}^{r} \frac{\frac{k}{3} r^{\prime} T_{0}^{0}(t)}{1-\frac{k}{3} r^{\prime 2} T_{0}^{0}(t)} d r^{\prime}$,

hence,

$g_{00}(r, t)=\left[1-\frac{2 G M}{c^{2} R_{o}(t)}\right] \sqrt{\frac{1-\frac{k}{3}\left[R_{o}(t)\right]^{2} T_{0}^{0}(t)}{1-\frac{k}{3} r^{2} T_{0}^{0}(t)}}$.

If $T_{0}^{0}$ is considered uniform, then (A.1) is simply

$g_{11}(r, t)=\frac{-1}{1-\frac{k}{3} r^{2} T_{0}^{0}(t)}$

Substituting (26) into (A.8) and (A.9), we get $g_{00}(r, t)=\left[1-\frac{k M c^{2}}{4 \pi R_{o}(t)}\right] \sqrt{\frac{1-\frac{k M c^{2}}{4 \pi R_{o}(t)}}{1-\frac{k M c^{2} r^{2}}{4 \pi\left[R_{o}(t)\right]^{3}}}}$,

$g_{11}(r, t)=\frac{-1}{1-\frac{k M c^{2} r^{2}}{4 \pi\left[R_{o}(t)\right]^{3}}}$,

and substituting (A.10) and (A.11) into (36), we get

$$
\begin{aligned}
\nabla^{i} \nabla_{i}^{E} h^{\prime}\left(k T_{0}^{0}\right) \cong & \frac{k}{8 \pi} \frac{1}{\left(1-\frac{k M c^{2}}{4 \pi R_{o}(t)}\right)^{3 / 2}} \frac{\partial h^{\prime}\left(k T_{0}^{0}\right)}{\partial t} \\
& \times \frac{r^{2}}{\sqrt{1-\frac{k M c^{2} r^{2}}{4 \pi\left[R_{o}(t)\right]^{3}}}} \frac{\partial}{\partial t} \frac{M}{\left[R_{o}(t)\right]^{3}} \\
= & \frac{k}{8 \pi} \frac{1}{\left(1-\frac{k M c^{2}}{4 \pi R_{o}(t)}\right)^{3 / 2}} \frac{\partial\left(k T_{0}^{0}\right)}{\partial t} h^{\prime \prime}\left(k T_{0}^{0}\right) \\
& \times \frac{r^{2}}{\sqrt{1-\frac{k M c^{2} r^{2}}{4 \pi\left[R_{o}(t)\right]^{3}}}} \frac{\partial}{\partial t} \frac{M}{\left[R_{o}(t)\right]^{3}} .
\end{aligned}
$$

Then, from (26) and (A.12), it follows

$$
\begin{aligned}
\nabla^{i} \nabla_{i}^{E} h^{\prime}\left(k T_{0}^{0}\right) \cong & \frac{3 k^{2} c^{2}}{32 \pi^{2}} \frac{h^{\prime \prime}\left(k T_{0}^{0}\right)}{\left(1-\frac{k M c^{2}}{4 \pi R_{o}(t)}\right)^{3 / 2}} \\
& \times \frac{r^{2}}{\sqrt{1-\frac{k M c^{2} r^{2}}{4 \pi\left[R_{o}(t)\right]^{3}}}}\left[\frac{\partial}{\partial t} \frac{M}{\left[R_{o}(t)\right]^{3}}\right]^{2},
\end{aligned}
$$

therefore,

$$
\begin{aligned}
\int_{o}^{R_{o}(t)} \nabla^{i} \nabla_{i}^{E} h^{\prime}\left(k T_{0}^{0}\right) r^{2} d r \cong \frac{3 k^{2} c^{2}}{32 \pi^{2}}\left[\frac{\partial}{\partial t} \frac{M}{\left[R_{o}(t)\right]^{3}}\right]^{2} \\
\times \frac{h^{\prime \prime}\left(k T_{0}^{0}\right)}{\left(1-\frac{k M c^{2}}{4 \pi R_{o}(t)}\right)^{3 / 2}} \int_{o}^{R_{o}(t)} \frac{r^{4}}{\sqrt{1-\frac{k M c^{2} r^{2}}{4 \pi\left[R_{o}(t)\right]^{3}}}} d r .
\end{aligned}
$$

Denoting

$\xi^{2}(t)=\frac{k M c^{2}}{4 \pi\left[R_{o}(t)\right]^{3}}$

we re-write (A.14) as

$\int_{o}^{R_{o}(t)} \nabla^{i} \nabla_{i}^{E} h^{\prime}\left(k T_{0}^{0}\right) r^{2} d r \cong \frac{3 k^{2} c^{2}}{32 \pi^{2}}\left[\frac{\partial}{\partial t} \frac{M}{\left[R_{o}(t)\right]^{3}}\right]^{2}$ 


$$
\times \frac{h^{\prime \prime}\left(k T_{0}^{0}\right)}{\left\{1-\left[\xi(t) R_{o}(t)\right]^{2}\right\}^{3 / 2}} \int_{o}^{R_{o}(t)} \frac{r^{4}}{\sqrt{1-\xi^{2}(t) r^{2}}} d r .
$$

Finally, it is easy to see that

$$
\begin{aligned}
& \frac{r^{4}}{\sqrt{1-\xi^{2} r^{2}}}= \\
& \frac{1}{8 \xi^{5}} \frac{\partial}{\partial r}\left[3 \arcsin (\xi r)-\xi r\left(3+2 \xi^{2} r^{2}\right) \sqrt{1-\xi^{2} r^{2}}\right]
\end{aligned}
$$

thus,

$$
\int_{o}^{R_{o}(t)} \nabla^{i} \nabla_{i}^{E} h^{\prime}\left(k T_{0}^{0}\right) r^{2} d r \cong h^{\prime \prime}\left(k T_{0}^{0}\right)\left[\frac{\partial}{\partial t} \frac{M}{\left[R_{o}(t)\right]^{3}}\right]^{2} \alpha(t),
$$

with

$$
\begin{aligned}
\alpha(t)= & \frac{3 k^{2} c^{2} R_{0}(t)}{256 \pi^{2}[\xi(t)]^{4}}\left\{\frac{3}{\xi(t) R_{0}(t)} \arcsin \left[\xi(t) R_{0}(t)\right]\right. \\
& \left.-\left(3+2\left[\xi(t) R_{0}(t)\right]^{2}\right) \sqrt{1-\left[\xi(t) R_{0}(t)\right]^{2}}\right\} \\
& \times\left(1-\left[\xi(t) R_{0}(t)\right]^{2}\right)^{-3 / 2} .
\end{aligned}
$$

\section{References}

1. S. Weinberg, Gravitation and cosmology: Principles and applications of the general theory of relativity (Wiley, New York, 1972)

2. L.D. Landau, E.M. Lifshitz, The classical theory of fields, vol. 2 (Elsevier, Oxford, 1994)

3. B.P. Abbott et al., [LIGO Scientific and Virgo Collaborations], Observation of gravitational waves from a binary black hole merger. Phys. Rev. Lett. 116, 061102 (2016). arXiv:1602.03837 [gr-qc]

4. B.P. Abbott et al., [LIGO Scientific and Virgo Collaborations], GW170817: Observation of gravitational waves from a binary neutron star inspiral. Phys. Rev. Lett. 119, 161101 (2017). arXiv: 1710.05832 [gr-qc]

5. P.J.E. Peebles, Principles of physical cosmology (Princeton University Press, Princeton, New Jersey, 1993)

6. S. Weinberg, The cosmological constant problem. Rev. Mod. Phys. 61, 1-23 (1989)

7. A. De Felice, S. Tsujikawa, $\mathrm{f}(\mathrm{R})$ theories. Living Rev. Rel. 13, 3 (2010). arXiv:1002.4928 [gr-qc]

8. T.P. Sotiriou, V. Faraoni, $f(R)$ theories of gravity. Rev. Mod. Phys. 82, 451 (2010). arXiv:0805.1726 [gr-qc]

9. S. Capozziello, M. De Laurentis, Extended theories of gravity. Phys. Rept. 509, 167 (2011). arXiv:1108.6266 [gr-qc]
10. L. Amendola, D. Polarski, S. Tsujikawa, Are $f(R)$ dark energy models cosmologically viable? Phys. Rev. Lett. 98, 131302 (2007). arXiv:astro-ph/0603703

11. H. Wei, H.Y. Li, X.B. Zou, Exact cosmological solutions of $f(R)$ theories via Hojman symmetry. Nucl. Phys. B 903, 132 (2016)

12. H. Wei, H .Y. Li, X .B. Zou, Are $f(R)$ dark energy models cosmologically viable? Phys. Rev. Lett. 98, 131302 (2007). arXiv:astro-ph/0603703, arXiv:1511.00376

13. S. Nojiri, S.D. Odintsov, Modified gravity with negative and positive powers of the curvature: Unification of the inflation and of the cosmic acceleration. Phys. Rev. D 68, 123512 (2003). arXiv:hep-th/0307288

14. H. Liu, X. Wang, H. Li, Y. Ma, Distinguishing $\mathrm{f}(\mathrm{R})$ theories from general relativity by gravitational lensing effect. Eur. Phys. J. C 77(11), 723 (2017). arXiv: 1508.02647

15. Z. Amirabi, M. Halilsoy, S. Habib Mazharimousavi, Generation of spherically symmetric metrics in $\mathrm{f}(\mathrm{R})$ gravity. Eur. Phys. J. C 76(6), 338 (2016). arXiv: 1509.06967

16. D. Müller, V .C. de Andrade, C. Maia, M .J. RebouÃ ăas, A .F .F. Teixeira, Future dynamics in $\mathrm{f}(\mathrm{R})$ theories. Eur. Phys. J. C 75(1), 13 (2015). arXiv:1405.0768 [astro-ph.CO]

17. T. Multamaki, I. Vilja, Spherically symmetric solutions of modified field equations in $\mathrm{f}(\mathrm{R})$ theories of gravity. Phys. Rev. D 74, 064022 (2006). [astro-ph/0606373]

18. K. Kainulainen, J. Piilonen, V. Reijonen, D. Sunhede, Spherically symmetric spacetimes in $\mathrm{f}(\mathrm{R})$ gravity theories. Phys. Rev. D 76, 024020 (2007). arXiv:0704.2729 [gr-qc]

19. A. Shojai, F. Shojai, Some static spherically symmetric interior solutions of $f(R)$ gravity. Gen. Rel. Grav. 44, 211 (2012). arXiv:1109.2190 [gr-qc]

20. M. Sharif, H .R. Kausar, Dust static spherically symmetric solution in $f(R)$ gravity. J. Phys. Soc. Jpn. 80, 044004 (2011). arXiv:1102.4124 [physics.gen-ph]

21. L. Sebastiani, S. Zerbini, Static spherically symmetric solutions in F(R) gravity. Eur. Phys. J. C 71, 1591 (2011). arXiv:1012.5230 [gr-qc]

22. A.L. Erickcek, T.L. Smith, M. Kamionkowski, Solar System tests do rule out 1/R gravity. Phys. Rev. D 74, 121501 (2006). arXiv:astro-ph/0610483

23. E.V. Arbuzova, A.D. Dolgov, L. Reverberi, Spherically Symmetric solutions in $\mathrm{F}(\mathrm{R})$ gravity and gravitational repulsion. Astropart. Phys. 54, 44 (2014). arXiv:1306.5694 [gr-qc]

24. A. Stabile, The Post-Newtonian limit of $f(R)$-gravity in the harmonic gauge. Phys. Rev. D 82, 064021 (2010). arXiv:1004.1973 [gr-qc]

25. S. Capozziello, A. Stabile, A. Troisi, A General solution in the Newtonian limit of f(R)- gravity. Mod. Phys. Lett. A 24, 659 (2009). arXiv:0901.0448 [gr-qc]

26. L.D. Landau, E.M. Lifshitz, Mechanics, vol. 1 (Elsevier, Oxford, 1994)

27. B. Majumder, The perihelion precession of Mercury and the generalized uncertainty principle. arXiv:1105.2428 [gr-qc] and references therein

28. S. Gillessen, F. Eisenhauer, S. Trippe, T. Alexander, R. Genzel, F. Martins, T. Ott, Monitoring stellar orbits around the massive black hole in the Galactic center. Astrophys. J. 692, 1075 (2009). arXiv:0810.4674 [astro-ph] 